\title{
High-gain control without identification: a survey
}

\author{
Achim Ilchmann* ${ }^{1}$ and Eugene P. Ryan** 2 \\ ${ }^{1}$ Institute of Mathematics, Technical University Ilmenau, Weimarer Straße 25, \\ 98693 Ilmenau, Germany \\ 2 Department of Mathematical Sciences, University of Bath, Claverton Down, \\ Bath BA2 7AY, UK
}

Received 5 November 2007

Key words Output feedback, nonlinear systems, functional differential equations, transient behaviour, tracking, minimum phase, relative degree.

Three related but distinct scenarios for tracking control of uncertain systems are reviewed: asymptotic tracking, approximate tracking with prescribed asymptotic error bound, tracking with prescribed transient behaviour. A variety of system classes are considered, ranging from finite-dimensional linear minimum-phase systems to nonlinear, infinite-dimensional systems described by functional differential equations. These classes are determined only by structural assumptions, such as stable zero dynamics and known relative degree. The objective is a single (and simple) control structure which is effective for every member of the underlying system class: no attempt is made to identify the particular system being controlled.

(C) 2008 WILEY-VCH Verlag GmbH \& Co. KGaA, Weinheim

\section{Introduction}

We describe "universal" control structures that achieve prescribed performance (for example, asymptotic tracking of a reference signal) for every system belonging to some underlying class. We stress that, in the present survey, we deal only with those contributions wherein no attempt is made to identify the particular system being controlled. The only a priori information available to the controller is the system class, determined by some set of structural assumptions. In essence, control without identification is the distinguishing feature: this contrasts with the early development of adaptive control until the 1980s.

We, therefore, consider given a class of systems $\Sigma=\{S\}$ characterized by particular structural assumptions. As a linear prototype, consider the class of $m$-input $\left(u(t) \in \mathbb{R}^{m}\right), m$-output $\left(y(t) \in \mathbb{R}^{m}\right)$, linear systems of the form

$$
\left.\begin{array}{l}
\dot{x}(t)=A x(t)+B u(t), \quad x(0)=x^{0} \in \mathbb{R}^{n} \\
y(t)=C x(t)
\end{array}\right\}
$$

where $A \in \mathbb{R}^{n \times n}, B, C^{T} \in \mathbb{R}^{n \times m}$ are unknown but satisfy the following structural assumptions:

\footnotetext{
* Corresponding author: e-mail: achim.ilchmann@tu-ilmenau.de

** e-mail:epr@maths.bath.ac.uk
} 
- (strict relative degree $\rho$ and sign-definite high-frequency gain)

for some known $\rho \in \mathbb{N}, C A^{i} B=0$ for $i=1, \ldots, \rho-2$ and $C A^{\rho-1} B$ is either positive definite or negative definite (symmetry is not imposed),

- (minimum phase)

$$
\operatorname{det}\left[\begin{array}{cc}
s I-A & B \\
C & 0
\end{array}\right] \neq 0 \quad \text { for all } s \in \mathbb{C} \text { with } \operatorname{Re} s \geq 0
$$

The minimum phase assumption is equivalent to the condition that, if the input is such that the output vanishes identically, then the unobserved internal dynamics (the so-called zero dynamics) are exponentially stable: in the context of nonlinear systems, the notion of minimum phase has counterparts (see, for example, [27, Subsections 4.3 and 5.1]).

Also given is a class of reference signals $\mathcal{Y}_{\text {ref }}$, typically, all absolutely continuous functions which are bounded with essentially bounded derivative

$$
\mathcal{Y}_{\text {ref }}=W^{1, \infty}\left(\mathbb{R}_{+}, \mathbb{R}^{m}\right):=\left\{r: \mathbb{R}_{+} \rightarrow \mathbb{R}^{m} \text { is abs. cont. } \mid r, \dot{r} \in L^{\infty}\left(\mathbb{R}_{+}, \mathbb{R}^{m}\right)\right\},
$$

or, more restrictively, all solutions of $\dot{r}=M r$ for known $M \in \mathbb{R}^{m \times m}$ (in contexts wherein the internal model principle, see, e.g. [43, 8, 28, 41], is invoked).

The control objective is to design a single output feedback controller $R:\left(y, y_{\mathrm{ref}}\right) \mapsto u$ such that, for every system $S \in \Sigma$ and every reference signal $y_{\text {ref }} \in \mathcal{Y}_{\text {ref }}$, all signals of the closedloop system $(S, R)$ are defined on $[0, \infty)$ and are bounded, and one of the following tracking objectives for the error

$$
e(t)=y(t)-y_{\text {ref }}(t), \quad y_{\text {ref }} \in \mathcal{Y}_{\text {ref }}
$$

should be achieved:

$\left(\mathrm{TO}_{1}\right)$ asymptotic tracking, i.e. $\lim _{t \rightarrow \infty} e(t)=0$,

$\left(\mathrm{TO}_{2}\right) \lambda$-tracking or sometimes called "approximate tracking", i.e. for any prescribed $\lambda>0$, $\limsup _{t \rightarrow \infty}\|e(t)\| \leq \lambda$

$\left(\mathrm{TO}_{3}\right)$ prespecified transient behaviour, i.e. for some suitable prescribed function $\varphi$, the error function is required to satisfy $\|e(t)\| \leq 1 / \varphi(t)$ for all $t>0$.

Historically, and also systematically, these three tracking objectives have been investigated in three stages. Initial investigations considered the above-mentioned prototype class of linear, minimum-phase systems, in the restricted context of stabilization (equivalently, $\mathcal{Y}_{\text {ref }}=\{0\}$ ). In the relative-degree-one case $(\rho=1)$, this class has a fundamental property.

High-Gain Property: Let $(A, B, C)$ be a minimum-phase linear system of relative degree $\rho=1$, with positive-definite high-frequency gain $C B>0$. Then there exists $k^{*}>0$ such that, for every $k \geq k^{*}$, the control $u(t)=-k y(t)$ applied to (1) yields an exponentially stable 
closed-loop system.

In the case of the extended class of nonlinear, infinite-dimensional, systems considered later, a (weaker) counterpart of the high-gain property holds. The high-gain property forms the basis of many early approaches to adaptive control without identification. Ubiquitous is the adaptation law $\dot{k}(t)=\|y(t)\|^{2}$ in conjunction with the feedback $u(t)=-k(t) y(t)$ : in Section 2, we discuss the far-reaching consequences of this simple strategy.

In Section 3, we show how this strategy can be extended to the problem of $\lambda$-tracking, through the introduction of a modified gain adaptation of the form $\dot{k}(t)=\max \left\{\|e(t)\|^{2}-\lambda, 0\right\}$.

In Section 4, we introduce the concept of funnel control wherein the (non-monotone) gain function is adjusted by, e.g., $k(t)=[1-\varphi(t)\|e(t)\|]^{-1}$, where the function $\varphi$ determines the transient behaviour of the tracking error.

In Section 5, we briefly review several mainly biotechnological applications of $\lambda$-tracking and also of funnel control. We also consider input constraints and other constraints given by the specific application. Most of the theoretical results have been supported by simulations, and some results have been tested in practice.

The obstacle of higher relative degree is discussed in Section 6. There are relatively few contributions on this problem: moreover, the simplicity of the relative-degree-one control structure is lost.

Finally, in Section 7 we deal with the problem of robustness. Here, the question is whether the performance of an adaptive controller - designed for a system class $\Sigma$ - persists when applied to a system outside the class $\Sigma$, but "close" (in terms of the gap metric) to some member of the class. Surprisingly, the standard high-gain controller (3) is still applicable to a general linear systems of the form (1) provided it is close - in the gap metric - to a system belonging to the prototype linear class (that is, if it is close - in the gap metric - to a linear minimum-phase system with positive high-frequency gain).

\section{High-gain adaptive control}

After thirty years of adaptive control based on identification mechanisms to estimate system parameters, investigators addressed the question of necessary and sufficient conditions for stabilization of linear systems. In his famous contribution [32], Mårtensson showed that "the order of any stabilizing regulator is sufficient a priori information for adaptive stabilization". While such existential problems were investigated, other contributions were made to show that the adaptive control law

$$
u(t)=-k(t) y(t), \quad \dot{k}(t)=\|y(t)\|^{2}, \quad k(0)=k^{0}
$$

stabilizes any single-input, single-output system belonging to the class of minimum-phase, relative-degree-one, linear systems (1), with positive high-frequency gain $C B$. More precisely, (3) applied to any such system, with arbitrary initial data $x^{0} \in \mathbb{R}^{n}, k^{0} \in \mathbb{R}$, yields a closed-loop, initial-value problem with unique solution $(x, k)$ and with the properties

$$
\lim _{t \rightarrow \infty} x(t)=0 \quad \text { and } \quad \lim _{t \rightarrow \infty} k(t)=k^{\infty} \in \mathbb{R} .
$$


Note the striking simplicity of the controller (3) and its underlying idea: a monotone gain function $k(\cdot)$ coupled with the high-gain property of the underlying system class.

This idea arose in several seminal papers almost at the same time: [34], [42], [4], [31]. Subsequently, it was generalized, preserving its simplicity, to different system classes: multivariable systems [16], unknown sign of the high-frequency gain [35], nonlinear systems [38], discontinuous feedback strategies within the framework of differential inclusions [36, 37], infinite-dimensional systems [29], transient behaviour [33], tracking including an internal model [8, 28], to name but a few. A survey of this field is in [11] and the monograph [12], and, in an infinite-dimensional context, in [30].

From a theoretical point of view, the importance of these contributions lay in the structural insight they provided which led later to more practical controller designs, see Sections 3 and 4. From a practical point of view, the canonical high-gain adaptive controller (3) (and variations thereof) are of limited use due to the following shortcomings:

$\left(\mathrm{HG}_{1}\right)$ The systems to which it applies have relative degree 1.

$\left(\mathrm{HG}_{2}\right)$ The gain $t \mapsto k(t)$ is monotonically increasing. Although theoretically convergent, perturbations to the system cause the gain to grow.

$\left(\mathrm{HG}_{3}\right)$ The class of systems feasible to this control is essentially restricted to linear systems.

$\left(\mathrm{HG}_{4}\right)$ Modification of the simple structure (3) to encompass tracking requires an internal model: this complicates the control.

$\left(\mathrm{HG}_{5}\right)$ Transient behaviour is not addressed.

$\left(\mathrm{HG}_{6}\right)$ Small measurement noise on the output can lead to an unbounded gain (essentially driven by the variance of the noise).

\section{High-gain adaptive $\lambda$-control}

The issues $\left(\mathrm{HG}_{3}\right),\left(\mathrm{HG}_{4}\right)$, and $\left(\mathrm{HG}_{6}\right)$ can be resolved by weakening the control objective slightly. In the context of tracking, instead of asymptotic tracking, it is required that all signals of the closed-loop system are bounded and the error $e$ approaches a ball of prespecified radius $\lambda>0$ : $\lim _{t \rightarrow \infty} \operatorname{dist}(\|e(t)\|,[0, \lambda])=0$; i.e., tracking objective $\left(\mathrm{TO}_{2}\right)$. We refer to this performance as $\lambda$-tracking or approximate tracking. To achieve $\lambda$-tracking for linear systems, the gain adaptation in (3) is modified to

$$
\left.\begin{array}{ll}
u(t)=-k(t) e(t), & e(t)=y(t)-y_{\mathrm{ref}}(t) \\
\dot{k}(t)=\max \{\|e(t)\|-\lambda, 0\}, & k(0)=k^{0} .
\end{array}\right\}
$$

In contrast to (3), the gain in (4) is constant on any interval in which the error is within the ball of radius $\lambda>0$. This minor modification (a) permits application to a large class of nonlinear systems since 0 is no longer required to be an equilibrium point and (b) obviates the necessity 
of an internal model but instead encompasses the large class of reference signals $\mathcal{Y}_{\text {ref }}$ given in (2).

In [1] and [13], nonlinear multi-input multi-output systems of the following form are considered:

$$
\left.\begin{array}{l}
\dot{y}=f(t, y, z)+g(t, y, z) u(t) \\
\dot{z}=h(t, y, z)
\end{array}\right\}
$$

with $f\left(t, y_{e}, z_{e}\right)=0$ and $h\left(t, y_{e}, z_{e}\right)=0$ for all $t$ (and so, with zero input $u=0,\left(y_{e}, z_{e}\right)$ is an equilibrium of the system), $g$ is uniformly bounded away from zero, and $\dot{z}=h\left(t, y_{e}, z\right)$ is uniformly asymptotically stable. In [1], the functions $f, g, h$ are assumed globally Lipschitz in $(y, z)$ uniformly in $t$ : in [13], only polynomial boundedness in $(y, z)$, uniformly in $t$, is assumed. In the latter case, in order to preclude finite escape times in the closed-loop system, an upper bound $s \geq 1$ of the polynomial degree of $f$ and $g$ is incorporated in the control law (4) as follows:

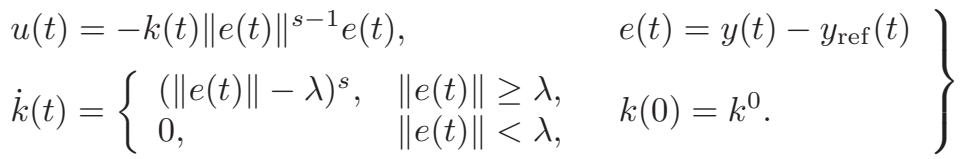

The tracking objective $\left(\mathrm{TO}_{2}\right)$ is achieved by applying (6) to any system of the form (5), satisfying the above-mentioned hypotheses.

A class related to (5) is that of infinite-dimensional, nonlinear $m$-input $u, m$-output $y$ systems given by a controlled functional differential equation

$$
\dot{y}(t)=f(p(t),(T y)(t))+g(p(t),(T y)(t), u(t)),\left.\quad y\right|_{[-h, 0]}=y^{0} \in C\left([-h, 0] ; \mathbb{R}^{m}\right)
$$

where, loosely speaking, $h \geq 0$ quantifies the "memory" of the system, $p$ may be thought of as a (bounded) disturbance term and $T$ is a nonlinear causal operator, $g$ and $f$ are only assumed to be continuous. Whilst a full description of this system class is given in [19], we remark here that diverse phenomena are incorporated within the class including, for example, diffusion processes, delays (both point and distributed) and hysteretic effects. Apart from some technical conditions, the essential hypotheses are (a) information on the growth of $f$, (b) the existence of some positive-definite, symmetric $G \in \mathbb{R}^{m \times m}$ such that

$$
u^{T} G g(p, w, u) \geq\|u\|^{2} \quad \forall(p, w, u) \in \operatorname{dom}(g) .
$$

(this assumption replaces the "positive high-frequency gain" assumption in the case of linear systems), and (c) a weak bounded-input, bounded-output assumption on the operator $T$ (which is a counterpart of the minimum-phase assumption in the case of linear systems). The feedback and gain adaptation takes the form

$$
u(t)=-k(t) \varphi(e(t)), \quad \dot{k}(t)=\psi_{\lambda}(e(t)), \quad k(0)=k^{0}
$$

where the functions $\varphi$ and $\psi_{\lambda}$ are determined by the hypotheses (a) and (c), alluded to above, together with the tracking accuracy parameter $\lambda>0$. The overall closed-loop system is 


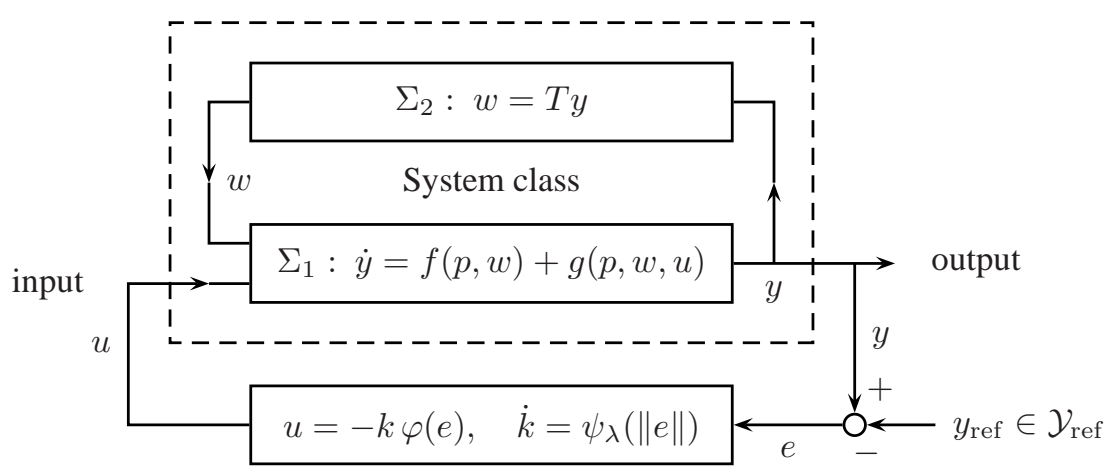

Fig. 1 Closed-loop $\lambda$-control system.

depicted in Figure 3.1. Note that (6) is a particular case of this general structure. The concept of $\lambda$-tracking is implicit in [33], albeit is a somewhat different context to that considered here. The concept as described above was introduced for the linear class (1) in [18], for infinitedimensional linear systems in [15], for the nonlinear class (1) in [1, 13], for the nonlinear class (7) in [19], and, for systems modelled by differential inclusions in [38]. Discussion on contributions to systems of higher relative degree are postponed until Section 6.

Whilst we have now addressed the shortcomings $\left(\mathrm{HG}_{3}\right),\left(\mathrm{HG}_{4}\right)$, and $\left(\mathrm{HG}_{6}\right)$, the following questions remain.

$\left(\lambda_{1}\right)$ How to tackle systems of relative degree $\rho \geq 2$ ?

$\left(\lambda_{2}\right)$ How to counteract the disadvantages of monotonically non-decreasing gain (which, whilst theoretically convergent, is susceptible to unwarranted increase generated by perturbations to the system)?

$\left(\lambda_{5}\right)$ How to influence transient behaviour?

\section{Funnel control}

To resolve $\left(\lambda_{2}\right)$ and $\left(\lambda_{5}\right)$, we first make the following observation. Loosely speaking, the high-gain property, alluded in the Introduction, ensures that the output $y(t)$ or the error $e(t)$ is decaying if the gain is sufficiently large. If $k$ is a time-varying function, we may "tune" its values $k(t)$ to be large only when required: $k$ need not be a monotonically increasing function.

With this in mind and to address the issue of transient behaviour, we introduce the concept of a performance funnel. Let $\varphi$ be a function of the following class

$$
\Phi:=\left\{\varphi \in W^{1, \infty}\left(\mathbb{R}_{+}, \mathbb{R}\right) \mid \varphi(0)=0, \varphi(s)>0 \text { for all } s>0 \text { and } \liminf _{s \rightarrow \infty} \varphi(s)>0\right\} .
$$


With $\varphi \in \Phi$, we associate the set

$$
\mathcal{F}_{\varphi}:=\left\{(t, e) \in \mathbb{R}_{+} \times \mathbb{R}^{m} \mid \varphi(t)\|e\|<1\right\},
$$

which we refer to as the performance funnel, see Figure 4.1. This terminology arises from the fact that, if a control structure can be devised which ensures that the tracking error evolves within $\mathcal{F}_{\varphi}$, then we have guaranteed transient behaviour (control objective $\left(\mathrm{TO}_{3}\right)$ ) in the sense that

$$
\|e(t)\|<1 / \varphi(t) \quad \forall t>0
$$

and, moreover, if $\varphi$ is chosen so that $\varphi(t) \geq 1 / \lambda$ for all $t$ sufficiently large, then control objective $\left(\mathrm{TO}_{2}\right)$ is achieved.

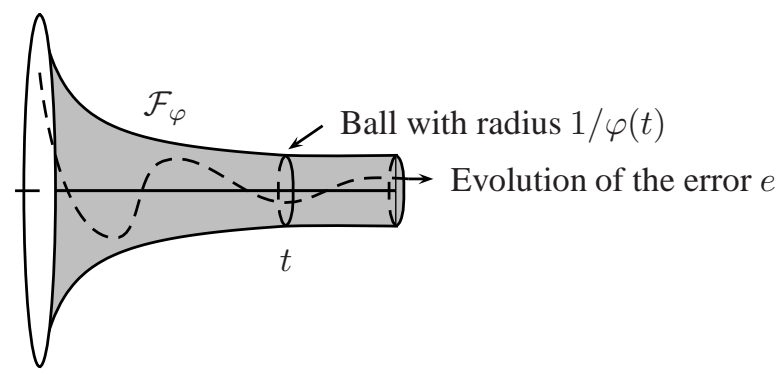

Fig. 2 Performance funnel $\mathcal{F}_{\varphi}$.

To ensure error evolution within the funnel, the controller (4) is replaced by

$$
\left.\begin{array}{l}
u(t)=-k(t) e(t), \quad e(t)=y(t)-y_{\mathrm{ref}}(t) \\
k(t)=[1-\varphi(t)\|e(t)\|]^{-1} .
\end{array}\right\}
$$

In view of the high-gain property, intuitively we see that, in order to maintain the error evolution within the funnel, high gain values may only be required when the error is close to the funnel boundary. This intuition underpins the choice of the gain function in (8). The structure (8) has two advantages: $k(t)$ is not determined by a dynamical system (differential equation); the control (8) is a time-varying proportional output feedback of striking simplicity. This structure was introduced for the class of nonlinear systems (7) in [20]: modifications to mollify controller behaviour near the funnel boundary are contained in [23].

\section{Applications}

Whereas there are few applications of the high-gain control approach described in Section 2, the $\lambda$-tracking approach has found several applications (including experimental implementation in the regulation of the $\mathrm{pH}$-value of a biogas tower reactor [17]).

Theoretical results and simulations for mostly biotechnological applications of $\lambda$-tracking are 
as follows: in anesthesia in [3]; for continuous stirred tank reactors in [1, 6]; for exothermic chemical reactors under input constraints in [24]; for chemical reaction models with sampleddata in [25]; for activated sludge processes in [14].

Funnel control under input constraints for chemical reactor models has been investigated in [26].

Recently, funnel control has been applied to electrical devices [39, 40] modelled as a two mass system, and the aim is to control the speed of the load mass in the presence of load disturbances and sensor noise.

\section{The obstacle of higher relative degree}

If a (linear) system has a higher relative degree and derivative feedback is not feasible, then a filter or observer is frequently used to obtain approximations of the output derivatives. However, it is not straightforward to determine how to combine a filter/observer with a high-gain controller.

A first attempt to achieve stabilization for linear systems of higher relative degree is due to Mareels [31]; however, a counterexample to the main result is presented in [9]. Unless otherwise stated, all results cited below relate to single-input, single-output systems. Hoagg and Bernstein $[9,10]$ solve the problem of adaptive stabilization of linear systems with higher relative degree. Bullinger and Allgöwer [2] introduce a high-gain observer in conjunction with an adaptive controller to ensure tracking with prescribed asymptotic accuracy $\lambda>0$ ( $\lambda$-tracking). This is achieved for a class of systems which are affine in the control, of known relative degree, and with affine linearly-bounded drift term; so they are close to linear. Paper [44] considers linear minimum-phase systems with nonlinear perturbation; the control objective is (continuous) adaptive $\lambda$-tracking with non-decreasing gain; the class of allowable nonlinearities is considerably smaller than that encompassed by (7). Stabilization for systems of maximum relative degree in the so-called parametric strict feedback form is achieved in [45] via a piecewise constant adaptive switching strategy. Both these contributions use a backstepping procedure.

The concept of funnel control is applied to linear systems of known relative degree in [21] and generalized to a class nonlinear systems (7) in [22]. However, in both contributions, a backstepping procedure is used which complicates the feedback structure. An alternative approach might be to combine a simple high-gain observer with a funnel-type controller.

\section{Robustness}

As we have seen, high-gain control is applicable to large classes of nonlinear systems. However, if a system of the underlying class is subjected to perturbations which take it outside the class, then it is not clear if the controller continues to maintain performance. This problem of robustness has been investigated for the class of linear systems (1).

In particular, exploiting the concept of the nonlinear gap metric (graph topology) (see, e.g. [7]) from the theory of robust stability, in [5] it is shown that the canonical high-gain controller (3) maintains its efficacy in the presence of $L^{2}$-input and $L^{2}$-output disturbances. It is 
proved that the requisite performance also persists when the plant $P=(A, B, C)$ is replaced by a stabilizable and detectable linear plant $P_{1}$ within a sufficiently small neighbourhood of $P$ in the graph topology, provided that the plant initial data and the $L^{2}$ magnitude of the disturbances are sufficiently small. Unstable behaviour for large initial conditions and/or large $L^{2}$ disturbances is shown, demonstrating that the bounds obtained from the $L^{2}$ theory are qualitatively tight.

The importance of these contributions is the result that $P_{1}$ is only assumed to be close to a system of the prototype linear class (1): $P_{1}$ need not be minimum phase and could have higher relative degree $\rho \geq 2$ (for examples, see the Introduction in [5]). This result supports the intuition of many engineers that certain subsystems may be neglected in system modelling as long as their contribution is sufficiently small in the gap metric sense. Robustness in the contexts of $\lambda$-tracking and funnel control remains an open question.

\section{References}

[1] F. Allgöwer, J. Ashman, and A. Ilchmann. High-gain adaptive $\lambda$-tracking for nonlinear systems. Automatica, 33:881-888, 1997.

[2] E. Bullinger and F. Allgöwer. Adaptive $\lambda$-tracking for nonlinear higher relative degree systems. Automatica, 41:1191-1200, 2005.

[3] E. Bullinger, C. Frei, T. Sieber, A.H. Glattfelderv F. Allgöwer, and A.M. Zbinden. Adaptive $\lambda$-tracking in anesthesia. In Proc. 4th IFAC Symposium Modelling and Control in Biomedical Systems, pages 181-186, Greifswald, 2000.

[4] C.I. Byrnes and J.C. Willems. Adaptive stabilization of multivariable linear systems. In Proc. 23rd IEEE Conf. on Decision and Control, pages 1574-1577, Las Vegas, Nevada, 1984.

[5] M. French, A. Ilchmann, and E.P. Ryan. Robustness in the graph topology of a common adaptive controller. SIAM J. of Control and Optim., 45(5):1736-1757, 2007.

[6] P. Georgieva, A. Ilchmann, and M.-F. Weirig. Modelling and adaptive control of aerobic continuous stirred tank reactors. European J. of Control, 7:476-491, 2001.

[7] T.T Georgiou and M.C. Smith. Robustness analysis of nonlinear feedback systems: an input-output approach. IEEE Trans. Aut. Control, 42:1200-1221, 1997.

[8] U. Helmke, D. Prätzel-Wolters, and S. Schmid. Adaptive tracking for scalar minimum phase systems. In D. Hinrichsen \& B. Mårtensson, editor, Control of Uncertain Systems, pages 101-117. Birkhäuser, Boston, 1990.

[9] J. B. Hoagg and D. S. Bernstein. Direct adaptive dynamic compenation for minimum phase systems with unknown relative degree. In Proc. 43rd IEEE Conf. on Decision and Control, pages 183-188, Paradise Island, The Bahamas, 2004.

[10] J. B. Hoagg and D. S. Bernstein. Direct adaptive command following and disturbance rejection for minimum phase systems with unknown relative degree. In Proc. 44th IEEE Conf. on Decision and Control, pages 2242-2247, Seville, Spain, 2005.

[11] A. Ilchmann. Non-identifier-based adaptive control of dynamical systems: a survey. IMA Journal of Math. Control and Inf., 8:321-366, 1991.

[12] A. Ilchmann. Non-Identifier-Based High-Gain Adaptive Control. Springer-Verlag, London, 1993.

[13] A. Ilchmann. Adaptive $\lambda$-tracking for polynomial minimum phase systems. Dynamics and Stability of Systems, 13:341-371, 1998.

[14] A. Ilchmann and P. Georgieva. Adaptive $\lambda$-tracking control of activated sludge processes. Int. J. of Control, 74:1247-1259, 2001.

[15] A. Ilchmann and H. Logemann. Adaptive $\lambda$-tracking for a class of infinite-dimensional systems. Sys. Control Lett., 34:11-21, 1998. 
[16] A. Ilchmann, D.H. Owens, and D. Prätzel-Wolters. High gain robust adaptive controllers for multivariable systems. Sys. Control Lett., 8:397-404, 1987.

[17] A. Ilchmann and M. Pahl. Adaptive multivariable regulation of a biogas tower reactor. European Journal of Control, 4:116-131, 1998.

[18] A. Ilchmann and E.P. Ryan. Universal $\lambda$-tracking for nonlinearly perturbed systems in the presence of noise. Automatica, 30:337-346, 1994.

[19] A. Ilchmann, E.P. Ryan, and C.J. Sangwin. Systems of controlled functional differential equations and adaptive tracking. SIAM J. of Control and Optim., 40:1746-1764, 2002.

[20] A. Ilchmann, E.P. Ryan, and C.J. Sangwin. Tracking with prescribed transient behaviour. ESAIM Control, Opt. and Calculus of Variations, 7:471-493, 2002.

[21] A. Ilchmann, E.P. Ryan, and P. Townsend. Tracking control with prescribed transient behaviour for systems of known relative degree. Sys. Control Lett., 55:396-406, 2006.

[22] A. Ilchmann, E.P. Ryan, and P. Townsend. Tracking with prescribed transient behaviour for nonlinear systems of known relative degree. SIAM J. of Control and Optim. to appear, 2007.

[23] A. Ilchmann, E.P. Ryan, and S. Trenn. Tracking control: performance funnels and prescribed transient behaviour. Sys. Control Lett., 54:655-670, 2005.

[24] A. Ilchmann, M. Thuto, and S. Townley. Input constrained adaptive tracking with applications to exothermic chemical reaction models. SIAM J. Control and Optim., 43:154-173, 2004.

[25] A. Ilchmann, M. Thuto, and S. Townley. Adaptive sampled-data tracking for input constrained exothermic chemical reaction models. Sys. Control Lett., 54:1149-1161, 2005.

[26] A. Ilchmann and S. Trenn. Input constrained control with applications to chemical reactor models. Sys. Control Lett., 53:327-424, 2004.

[27] A. Isidori. Nonlinear Control Systems. Springer-Verlag, London, 3 edition, 1995.

[28] H. Logemann and A. Ilchmann. An adaptive servomechanism for a class of infinite-dimensional systems. SIAM J. of Control and Optim., 32:917-936, 1994.

[29] H. Logemann and B. Mårtensson. Adaptive stabilization of infinite-dimensional systems. IEEE Trans. Aut. Control, 37:1869-1883, 1992.

[30] H. Logemann and S. Townley. Adaptive control of infinite-dimensional systems without parameter estimation: an overview. IMA J. of Math. Control and Inf., 14:175-206, 1997.

[31] I.M.Y. Mareels. A simple selftuning controller for stably invertible systems. Syst. Control Lett., 4:5-16, 1984

[32] B. Mårtensson. The order of any stabilizing regulator is sufficient a priori information for adaptive stabilization. Syst. Control Lett., 6:87-91, 1985.

[33] D.E. Miller and E.J. Davison. An adaptive controller which provides an arbitrarily good transient and steady-state response. IEEE Trans. Aut. Control, 36(1):68-81, 1991.

[34] A.S. Morse. Recent problems in parameter adaptive control. In I.D. Landau, editor, Outils et Modèles Mathématiques pour l'Automatique, l'Analyse de Systèmes et le Traitment du Signal, pages 733-740. Birkhäuser Verlag, 1983.

[35] R.D. Nussbaum. Some remarks on a conjecture in parameter adaptive control. Syst. Control Lett., 3:243-246, 1983.

[36] E.P. Ryan. Adaptive stabilization of a class of uncertain nonlinear systems: A differential inclusion approach. Syst. Control Lett., 10:95-101, 1988.

[37] E.P. Ryan. Universal $W^{1, \infty}$-tracking for a class of nonlinear systems. Syst. Control Lett., 18:201210, 1992

[38] E.P. Ryan. A nonlinear universal servomechanism. IEEE Trans. Aut. Control, 39:753-761, 1994.

[39] H. Schuster, C. Hackl, C. Westermaier, and D. Schröder. Funnel-control for electrical drives with uncertain parameters. In Proc. 7th IEEE Int. Power Engg. Conf., IPEC, Singapore, 2005.

[40] H. Schuster, C. Westermaier, and D. Schröder. Non-identifier-based adaptive control for a mechatronic system achieving stability and steady state accuracy. In Proc. 2006 IEEE Int. Conf. on Control Applications, CCA, pages 1819-1824, Munich, Germany, 2006.

[41] E.D. Sontag. Adaptation and regulation with signal detection implies internal model. Syst. Control Lett., 50:119-126, 2003. 
[42] J.C. Willems and C.I. Byrnes. Global adaptive stabilization in the absence of information on the sign of the high frequency gain. In A. Bensoussan and J.L. Lions, editors, Analysis and Optimization of Systems, Proc. of the 6th INRIA Conference, Nice, France, volume 62 of Lecture Notes in Control and Information Sciences, pages 49-57. Springer-Verlag, Berlin, 1984.

[43] W.M. Wonham. Linear Multivariable Control: a Geometric Approach. Springer-Verlag, New York - Heidelberg - Berlin, 1979.

[44] X. Ye. Universal $\lambda$-tracking for nonlinearly-perturbed systems. Automatica, 35:109-119, 1999.

[45] X. Ye. Switching adaptive output-feedback control of nonlinearly parameterized systems. Automatica, 41:983-989, 2005. 